\title{
Materials and Microfabrication Processes for ARROW-based Optofluidic Biosensors
}

\author{
Thomas Wall $^{1 *}$, Joshua Parks ${ }^{2}$, Holger Schmidt ${ }^{2}$, and Aaron R. Hawkins ${ }^{1}$ \\ ${ }^{1}$ Department of Electrical and Computer Engineering, Brigham Young University, Provo, UT 84602 \\ ${ }^{2}$ University of California, Santa Cruz, 1156 High St, Santa Cruz, CA 95064, U.S.A. \\ *thomas.wall@byu.edu
}

\begin{abstract}
This paper outlines the microfabrication processes and materials used to make an optofluidic lab-on-a-chip biosensor that detects individual biological particles. The biosensor uses a hollow-core ARROW waveguide with a low refractive index liquid core and is fabricated on a silicon wafer using a combination of PECVD deposition, RIE etching, and standard photolithographic processes. As a sensing example, detection of fluorescence signals emitted by labeled oligonucleotides inside the liquid core was used to illustrate the chip's potential to identify protein-coding regions of the Zaire Ebola virus genome.
\end{abstract}

\section{INTRODUCTION}

Optofluidic platforms, which combine optics and microfluidics in a single system, have recently become popular for use as biosensors [1]. There exists a wide variety of techniques and methods that can be employed in order to create an optical biosensor. Some of these methods include interferometry, evanescent fields, fluorescence, and spectral absorption $[1,2]$. We present an optofluidic waveguiding labon-a-chip that uses a hollow-core anti-resonant reflecting optical waveguide (ARROW) in order to detect individual biological particles [3]. This ARROW biosensor uses intersecting planar waveguides to direct light to miniscule interaction volumes of a liquid sample. The biosensor detects particles within the liquid sample by making use of fluorescent dye markers. These fluorophores can be selectively attached to a particle in question, resulting in a fluorescence burst as the labeled particles pass by an excitation point. Fluorescence signals can be distinguished from excitation signals allowing for detection and counting of labeled particles [3].

The amount of light given off by a single fluorophore is small, making it important that the biosensor have a high efficiency for directing fluorescence signals to photodetectors. It is a given that any material or fabrication process choices must be made with this requirement in mind. This paper outlines the fabrication process for these biosensors and the reasoning behind the materials and steps used in order to maximize the sensitivity of the biosensor.

\section{ARROW BIOSENSOR}

The ARROW biosensor is designed to be capable of single bioparticle detection. Figure 1 shows the general structure, which includes a hollow-core anti-resonant reflective optical waveguide (ARROW) capable of guiding light through a liquid filled low refractive index core. Intersecting the hollow-core is a solid-core ridge waveguide [4]. These solid-core waveguides allow for light to be coupled on and off the chip via butt coupling with fiber optics. Two reservoirs are attached over the openings of the hollow-core ARROW waveguide to allow a liquid sample to be introduced to the hollow core ARROW waveguide from large macrointerfaces.

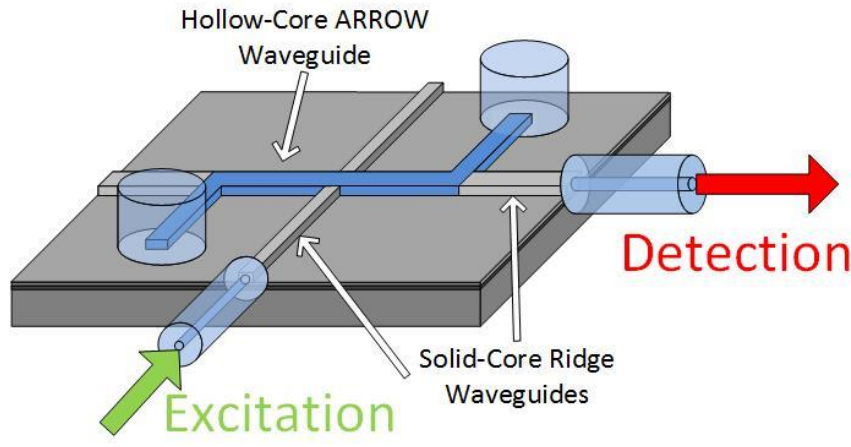

Figure 1: Schematic of the optofluidic biosensor.

In order to detect nucleic acids within a sample, a sample is first pre-treated by specific labeling with a fluorophore. Any labeled particles will fluoresce when excited by a specific wavelength of light. Examples of popular fluorophores can be excited by a $488 \mathrm{~nm}$ Argon laser or a $633 \mathrm{~nm}$ HeNe laser [5]. After labeling, the sample is introduced into the sensor through one of the attached reservoirs and an external pressure is applied that pushes the sample through the hollow core ARROW waveguide. Laser light coupled into the intersecting solid-core ridge waveguide passes through the hollow core ARROW waveguide and excites any marked particles that flow by it. The generated fluorescence is guided down the ARROW waveguide and off chip via a butt coupled optical fiber or a collection objective lens. The signal is then carried to a single photon avalanche photodiode (SPAPD). Any signal above the noise floor detected by the SPAPD represents a selectively marked bioparticle being present in the sample. 


\section{FABRICATION PROCESS}

The biosensor has been developed over time in order to maximize its sensitivity. This section discusses the fabrication methods that are currently used and represents the most up-todate methods. Also discussed are the materials that are used in fabrication.

The entire fabrication process for the biosensor consists of 6 major steps that are shown below in Figure 2.

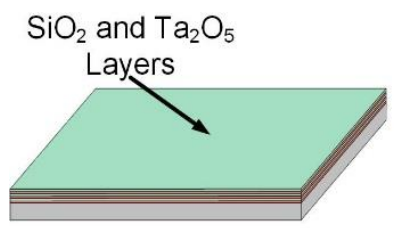

(a)

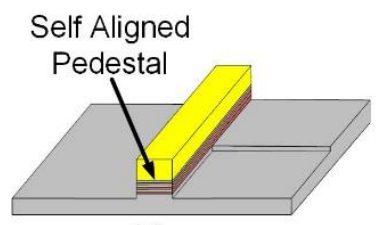

(c)

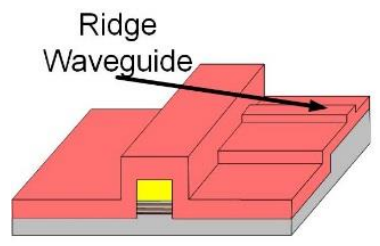

(e)

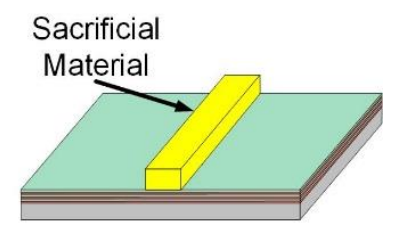

(b)

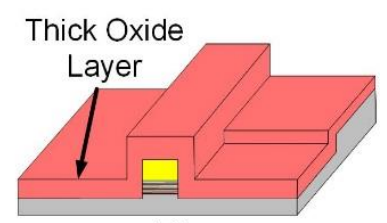

(d)

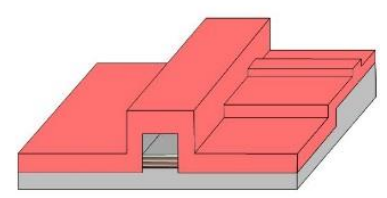

(f)
Figure 2: Major steps in the fabrication process for the ARROW biosensor. (a) Alternating stack of $\mathrm{SiO}_{2}$ and $\mathrm{Ta}_{2} \mathrm{O}_{5}$ is deposited via sputtering. (b) Sacrificial core for ARROW waveguide is deposited and patterned using photolithography. (c) Self aligned pedestal (SAP) is etched using RIE etching. (d) Thick oxide layer is deposited over the top of the sacrificial core using PECVD. (e) Standard ridge waveguides etched into $\mathrm{SiO}_{2}$ top layer to allow for coupling into ARROW waveguide. (f) Sacrificial core is etched away using a piranha acid mixture.

\section{Deposit Bottom ARROW Layers}

The ARROW waveguide in our biosensor makes use of a dielectric stack to create interference and guide light through a low refractive index core [4]. We use a stack of 6 alternating $\mathrm{SiO}_{2}$ and $\mathrm{Ta}_{2} \mathrm{O}_{5}$ layers [6]. The layers are deposited on the silicon substrate via sputtering.

The materials used in the dielectric stack must have low photoluminescence in the visible wavelength range. This is due to the fact that any luminescence from these layers will simply add to the background noise of the biosensor and lower its sensitivity. $\mathrm{Ta}_{2} \mathrm{O}_{5}$ and $\mathrm{SiO}_{2}$ were both determined to have lower photoluminescence in the visible range than other potential cladding layers [6].

\section{Deposit and Pattern the Sacrificial Core}

SU-8 (a commonly used negative photoresist) is spun onto the wafer and patterned into cores using standard photolithographic techniques. The cores are used as a sacrificial material for subsequent surface micromachining. SU-8 photoresist was chosen for three main reasons: its rectangular shape after developement, its ability to maintain this shape at higher temperatures, and its available selective etch.

The rectangular shape of the hollow core is important because it allows for efficient coupling between the ridge and ARROW waveguides. A rectangular shape creates a clean planar surface for interfacing between the two waveguides and maximizes the throughput of the light at this intersection.

Many different photoresists were tested in order to determine if they could maintain a rectangular shape at temperatures around $300^{\circ} \mathrm{C}$. Most of the photoresists would either crack or reflow, which rounded out the shape of the core. However, SU-8 does not crack and maintains an approximately rectangular shape.

SU-8 has an available selective etch with $\mathrm{SiO}_{2}$ which allows for the successful etch removal of the sacrificial core in step 6. A common acid mixture, called piranha, can be used to dissolve the SU-8 and does not attack the structural top dielectric layers of the ARROW waveguide.

\section{Self-Aligned Pedestal}

The biosensor's sensitivity depends heavily on the propagation loss in the hollow core ARROW waveguide. One method of minimizing this propagation loss is to surround the waveguide with a low refractive index material. The ARROW waveguide should be surrounded by air (Air has the lowest achieveable refractive index of, $n=1$ ) as much as possible in order to lower its propagation loss [7].

To surround the ARROW waveguide's sidewalls with air the core should sit on top of a pedestal that raises it above the surface. The pedestal is created using a process that we call a self-aligned pedestal (SAP) [8]. The sacrificial core is first protected by a nickel over coating using a standard liftoff procedure. The bottom ARROW layers and silicon below are then etched using an RIE etcher and a deep, silicon etch recipe [9].

\section{Deposit Top ARROW Layers}

Next, a $6 \mu \mathrm{m}$ thick $\mathrm{SiO}_{2}$ layer is deposited over the wafer by plasma enhanced chemical vapor deposition (PECVD). This layer encloses the sacrificial core and will act as the structural layer of the ARROW waveguide after the core is etched away. This top ARROW layer is used to create the ridge waveguides that couple with the ARROW waveguide. Because light will guide in this layer many considerations must be made in order to ensure that the layer does not degrade the biosensor's sensitivity. Some of these considerations include what material to use, how to deposit the material, and how thick the layer should be.

$\mathrm{SiO}_{2}$ was chosen as the primary cladding material for several reasons. First, $\mathrm{SiO}_{2}$ has transmission in the visible range. Any loss in the top layer lowers the sensitivity of the biosensor. The next reason is that $\mathrm{SiO}_{2}$ holds up to the long sacrificial core etch. The layer must not etch in piranha acid and cannot crack during the aggressive removal of the 
sacrificial core. Finally, we chose $\mathrm{SiO}_{2}$ because of its low photoluminescence in the visible range, which helps lower the background noise in the biosensor.

The next major consideration is how to deposit the top ARROW layer. The deposition method of a thin film can greatly affect the material properties of the layer [10]. The top ARROW layer needs to be deposited at a relatively low temperature $\left(<300^{\circ} \mathrm{C}\right)$ in order to protect the device from damage during deposition.

There are only a few deposition methods that exist for the deposition of $\mathrm{SiO}_{2}$ at low temperatures. These include PECVD, sputter deposition, and spin-on deposition using a spin-on-glass (SOG). Theoretically, sputter deposition should yield a dense and conformal $\mathrm{SiO}_{2}$ film [10]; however, sputtered $\mathrm{SiO}_{2}$ tends to show structural failure during the sacrificial core etch. Spin-on glass deposition also failed to produce layers that could withstand the sacrificial core etch, leaving PECVD as our only viable deposition method.

The last parameter considered is the thickness of the top ARROW layer. Our biosensors were originally fabricated using a relatively thin top layer $(\sim 3 \mu \mathrm{m})$. We have found that a thicker top $\mathrm{SiO}_{2}$ layer $(\sim 6 \mathrm{~m})$ helps decrease optical loss within the biosensor.

A crevice forms near the hollow core ARROW waveguide during the deposition of the top layer because of poor step coverage. When PECVD is used to deposit over a rectangular topology an effect occurs called bread-loafing [11]. As a result, this crevice forms and its presence at the interface of the ridge and ARROW waveguides causes poor optical coupling efficiency. A thicker oxide helps shift the modes and align them, which increases the coupling efficiency at the interface. Figure 4 shows the results of a study on that shows an obvious increase in coupling efficiency with increasing thickness.

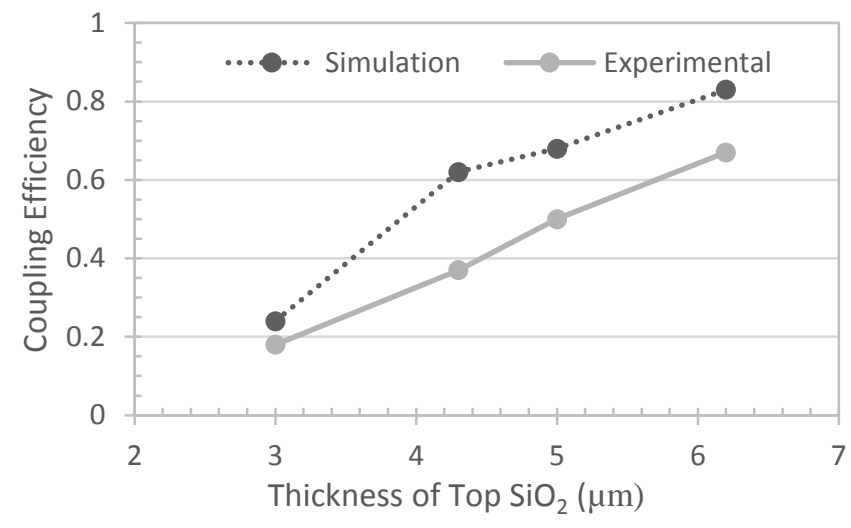

Figure 3: Simulation results with crevice (dots) and experimental results (solid) for couplinf efficiency at the solid to hollow core interface [6].

The top ARROW layer has some thickness limits because the quality of the PECVD $\mathrm{SiO}_{2}$ film decreases with thickness. One reason for this is the snowing effect in the PECVD chamber. During a deposition, $\mathrm{SiO}_{2}$ will coat the sample and also the chamber walls. As this layer on the chamber walls gets thicker it begins to crack and flake off. These flakes land on the sample and become defects in the $\mathrm{SiO}_{2}$ layer - defects that cause optical scattering in the waveguide. Note that optimal PECVD deposition conditions can be quite different for different systems and machines other than the one used in the cited study may be able to produce thicker high-quality films.

\section{Pattern and Etch the Solid-Core Ridge Waveguide}

The next fabrication step is to define the ridge waveguides. The ridge waveguide is made to be $4 \mu \mathrm{m}$ wide and $3 \mu \mathrm{m}$ tall to try and match its fundamental mode with the mode of a $633 \mathrm{~nm}$ single mode optical fiber. SU-8 was chosen as a mask material for this step because it has a good line resolution at high thicknesses. After the SU-8 mask is deposited and patterned the ridge waveguide is anisotropically etched into the $\mathrm{SiO}_{2}$ top layer using an RIE/ICP etcher. The sidewalls of the waveguide should be as straight and smooth as possible in order to minimize any optical loss in the waveguide.

\section{Sacrificial Core Etch}

The final fabrication step is to remove the sacrificial core, creating a hollow core ARROW waveguide. In order to etch away the sacrificial SU-8 material we must first expose the sacrificial core of the ARROW waveguide. A positive photoresist (AZ4620) mask used to protect the devices. The $\mathrm{SiO}_{2}$ layer over the sacrificial core's edges is then etched away using a BHF wet etch. With the core exposed the sacrificial SU-8 material is removed using an extended wet etch in a piranha acid mixture $\left(60 \mathrm{~mL}: 40 \mathrm{~mL}, \mathrm{H}_{2} \mathrm{O}_{2}: \mathrm{H}_{2} \mathrm{SO}_{4}\right)$ for several days [12].

\section{RESULTS}

As an example of our platform's sensing capabilities, it was used to selectively detect protein-coding regions of the Zaire Ebola virus genome (GeneBank ID AY354458.1) [5]. The results and procedure used for this detection are shown in Figure 5 and discussed below.

Target nucleic acids (100mer oligomers with a sequence specific to Zaire Ebola virus) were marked with a fluorescent dye. This was done by mixing the target nucleic acids with a fluorescent molecular beacon, see Figure 5(a), designed to attach only to the target nucleic acid. The target nucleic acid was then "pulled down" out of the solution by a biotinylated "pull-down" oligonucleotide attached to magnetic beads. Note, the pull-down oligonucheotide is also designed to only attach to the target nucleic acid, shown in Figure 5(a). The solution was then washed, to clear away anything that was not attached to the magnetic bead. The magnetic beads, with attached labeled nucleic acids, were then introduced to the biosensor.

The particles were pushed through the hollow core ARROW waveguide and passed by the detection point, where they were excited by $633 \mathrm{~nm}$ HeNe laser light and underwent fluorescence emission. The signal was then guided off-chip where it was detected by a SPAPD.

Figure 5(c) shows the signal detected when the target nucleic acid was present during the mixing step. Any peak represents a magnetic bead that passed by the detection point. Figure 5(b) 
shows the signal obtained with no target nucleic acids present during the mixing step. The magnetic beads that had no target nucleic acids attached gave off no signal. This demonstrates the ability of the ARROW optofluidic biosensor to selectively detect the presence of protein-coding regions of the Zaire Ebola virus.

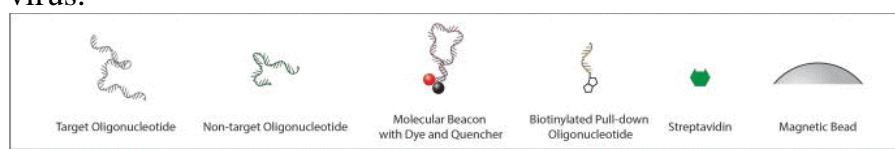

(a)
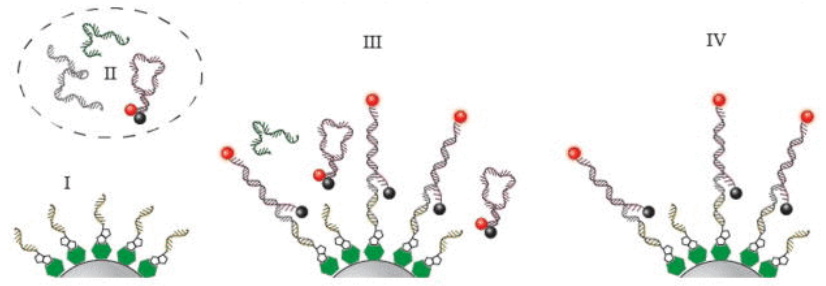

(b)

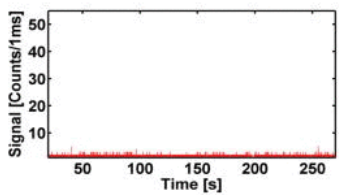

(c)

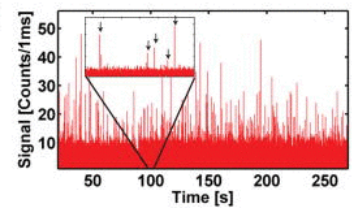

Figure 4: Detection of Zaire Ebola virus on-chip. (a) Nucleic acid labelling process, (I) magnetic beads and (II) nucleic acid target (Zaire Ebola virus) are mixed off chip (III); the resulting labelled particles (IV) are sent to the ARROW chip. (b) Signal with no target nucleic acids present during mixing. (c) Signal with presence of target nucleic acids, showing selective particle detection of Ebola viral sample, reprinted with permission from AIP: Biomicrofluidics [5].

In summary, we have designed and tested an optofluidic waveguiding lab-on-a-chip for detecting bioparticles. The biosensor makes use of a hollow core ARROW waveguide and intersecting solid core ridge waveguides in order to probe and excite particles in a small volume of a sample. We discussed the material considerations necessary to achieve high sensitivity detection. We also demonstrated the selective detection of protein-coding regions of the Zaire Ebola virus genome using our optofluidic biosensor.

\section{ACKNOWLEDGMENTS}

This work is supported by the NIH/NIAID and NSF under grants 1R21 AI100229, ECCS-1101801, ECCS-1101902, CBET-1159423, and CBET-1159453. J.W.P acknowledges support by the Eugene Cota-Robles Fellowship and the National Science Foundation Graduate Fellowship Research Program under Grant No. DGE 0809125. T.A.W. acknowledges support from the NASA Space Grant Consortium Fellowship. We would also like to thank the W.M. Keck Center at the University of California at Santa Cruz as well as the Integrated Microfabrication Laboratory at Brigham Young University.

\section{REFERENCES}

[1] H. Schmidt and A. R. Hawkins, "The photonic integration of non-solid media using optofluidics," Nature Photonics, vol. 5, pp. 598-604, Oct 2011.

[2] D. Erickson, S. Mandal, A. H. Yang, and B. Cordovez, "Nanobiosensors: optofluidic, electrical and mechanical approaches to biomolecular detection at the nanoscale," Microfluidics and nanofluidics, vol. 4, pp. 33-52, 2008.

[3] D. L. Yin, E. J. Lunt, M. I. Rudenko, D. W. Deamer, A. R. Hawkins, and H. Schmidt, "Planar optofluidic chip for single particle detection, manipulation, and analysis," Lab on a Chip, vol. 7, pp. 1171-1175, 2007. M. A. Duguay, Y. Kokubun, T. L. Koch, and L. Pfeiffer, "Antiresonant Reflecting Optical WaveGuides in Sio2-Si Multilayer Structures," Applied Physics Letters, vol. 49, pp. 13-15, Jul 71986.

J.W., Parks, et al., "Integration of programmable microfluidics and on-chip fluorescence detection for biosensing applications," AIP Biomicrofluidics, vol. 8(5), 2014.

[6] Y. Zhao, M. Jenkins, P. Measor, K. Leake, S. Liu, H. Schmidt, et al., "Hollow waveguides with low intrinsic photoluminescence fabricated with Ta2O5 and SiO2 films," Applied Physics Letters, vol. 98, Feb 282011.

[7] D. L. Yin, J. P. Barber, A. R. Hawkins, and H. Schmidt, "Waveguide loss optimization in hollowcore ARROW waveguides," Optics Express, vol. 13, pp. 9331-9336, Nov 142005.

[8] E. J. Lunt, B. Wu, J. M. Keeley, P. Measor, H. Schmidt, and A. R. Hawkins, "Hollow ARROW Waveguides on Self-Aligned Pedestals for Improved Geometry and Transmission," Ieee Photonics Technology Letters, vol. 22, pp. 1147-1149, Aug 1 2010.

[9] I. W. Rangelow, "Critical tasks in high aspect ratio silicon dry etching for microelectromechanical systems," Journal of Vacuum Science \& Technology A, vol. 21, pp. 1550-1562, Jul-Aug 2003.

[10] M. Ohring, Materials science of thin films: Academic press, 2001.

[11] G. C. Schwartz and K. V. Srikrishnan, Handbook of semiconductor interconnection technology: CRC Press, 2006.

[12] M. Holmes, J. Keeley, K. Hurd, H. Schmidt, and A. Hawkins, "Optimized piranha etching process for SU8-based MEMS and MOEMS construction," Journal of Micromechanics and Microengineering, vol. 20, Nov 2010. 